INVESTIGACIÓN

Recibido: 20/02/2019 --- Aceptado: 10/05/2019 --- Publicado: 15/12/2019

\title{
CHALLENGE BASED LEARNING COMO MODELO DE APRENDIZAJE PROFESIONALIZANTE. CASO DEL PROGRAMA UNIVERSIDAD EUROPEA CON COMUNICA +A
}

\section{Challenge Based Learning as a professional learning model. Universidad Europea and Comunica $+A$ program case study}

\begin{abstract}
(D) 87 María Mercedes Agüero Pérez. Universidad Europea de Madrid. España. mariamercedes.aguero@universidadeuropea.es
\end{abstract}

D (83) Luis Antonio López Fraile1. Universidad Europea de Madrid. España. luis.lopez@universidadeuropea.es

Javier Pérez Expósito. Universidad Europea de Madrid. España. javier.perez3@universidadeuropea.es

\section{RESUMEN}

El tradicional paradigma educativo, donde el profesor es centro del aprendizaje y proveedor de conocimiento, ha sido desplazado por nuevos métodos que ponen al alumno en el centro del proceso a través del aprendizaje experiencial, situándole ante casos y desafíos reales para que genere soluciones, donde el profesor pasa a ser un facilitador del proceso. Dentro de esta nueva visión se enmarca el Challenge Based Learning (CBL), centro de esta investigación que indaga acerca de si su utilización ayuda a mejorar los aspectos académicos, competenciales y de empleabilidad de los egresados en Publicidad. En esta línea de modernización de los procesos educativos, la facultad de CC. Sociales y de la Comunicación de la Universidad Europea de Madrid puso en marcha un programa basado en CBL en colaboración con la prestigiosa agencia de publicidad Comunica + A. Se trata de averiguar si esta metodología educativa es valorada e incorporada positivamente por los estudiantes. Para conseguirlo, se lleva a cabo una investigación consistente en una revisión bibliográfica y en un análisis descriptivo de los cuestionarios cumplimentados por los estudiantes participantes, para recabar su percepción sobre la utilidad y eficacia del programa en los aspectos

\footnotetext{
${ }^{1}$ Luis Antonio López Fraile: Doctor en Comunicación "Cum Laude" por la Universidad Europea de Madrid. Licenciado en CC. Políticas y Sociología por la Universidad Complutense de Madrid y Master en Dirección de RR.HH. y Organización por ESIC.
} 
Agüero Pérez, M. M.; López Fraile, L. A. y Pérez Expósito J. Challenge Based Learning como modelo de aprendizaje profesionalizante.

Caso del programa Universidad Europea con Comunica $+A$.

académicos, competenciales y de empleabilidad. Como conclusión, la hipótesis queda confirmada, con lo que se sientan las bases para un nuevo paradigma educativo (CBL) que permitirá adecuar las habilidades de los estudiantes a las nuevas necesidades del mercado.

PALABRAS CLAVE: aprendizaje basado en retos - coeducación - competencia profesional - aprendizaje activo - Universidad Europea - publicidad - empleabilidad competencias.

\section{ABSTRACT}

The traditional paradigm where the professor is the base of the learning and the only source of knowledge, it has been moved by new models that place the students in the center of the process through an experiential learning where they have to face realworld challenges where the teacher is just a provider during the process. This is the Challenge Based Learning (CBL) new framework, and the key point of this research is to prove if this model improves the academic results, skills and employability of our graduates in Advertising Communication Degree. Following this updating educational approach, Social Science and Communication School at European University of Madrid launched a program based on CBL in partnership with a prestigious advertising agency: Comunica +A. It is about finding out if this educational model is positively valued and accepted by the students. To achieve this goal, a research was developed consisting in a bibliography review and a descriptive analysis of the questionnaires fulfilled by the participants in the program. The objective was to collect the student's perception about the usefulness and effectiveness of the program regarding academic results, skills and employability. In conclusion, the hypothesis is confirmed and consequently the foundation for a new learning model (CBL) has been set. This model is aligning student's competences with the new market's needs.

KEY WORDS: challenge based learning - coeducation - professional competence active learning - Universidad Europea - advertising - employability - skills.

\section{CHALLENGE BASED LEARNING COMO MODELO DE APRENDIZAGEM PROFISSIONALIZANTE. CASO DO PROGRAMA UNIVERSIDADE EUROPEIA COM COMUNICA + A}

\section{RESUME}

O tradicional paradigma educativo, onde o professor é o centro da aprendizagem e provedor de conhecimento, foi deslocado por novos métodos que põem ao aluno no centro do processo através do aprendizado experimental, situando-os ante casos e desafios reais para que gere soluções, onde o professor passa a ser um facilitador do

Vivat Academia. Revista de Comunicación. 15 diciembre 2019 /15 marzo 2020, nº 149, 1-24 
Agüero Pérez, M. M.; López Fraile, L. A. y Pérez Expósito J. Challenge Based Learning como modelo de aprendizaje profesionalizante.

Caso del programa Universidad Europea con Comunica $+A$.

processo. Dentro desta nova visão enquadra o Challenge Based Learning (CBL), centro desta investigação que indaga sobre se sua utilização ajuda a melhorar os aspectos acadêmicos, habilidade dos graduados na Publicidade. Nesta linha de modernização dos processos educativos, a faculdade de CC Sociais e da Comunicação da Universidade Europeia de Madri colocou em andamento um programa baseado em CBL em colaboração com a prestigiosa agencia de publicidade Comunica $+\mathrm{A}$. Se trata de averiguar se esta metodologia educativa é valorizada e incorporada positivamente pelos estudantes. Para consegui-lo, se utiliza uma investigação consistente em uma revisão bibliográfica e em uma analises descritiva dos questionários concluídos pelos estudantes participantes, para coletar sua percepção sobre a utilidade e eficácia do programa nos aspectos acadêmicos, competências e de empregabilidade. Como conclusão, a hipóteses fica confirmada, com as bases assentadas, para um novo paradigma educativo (CBL) que permitirá adequar as habilidades dos estudantes as novas necessidades do mercado.

PALAVRAS CHAVE: aprendizagem baseado em retos - coeducação - habilidade profissional - aprendizagem ativo - Universidade Europeia - publicidade empregabilidade - competências - reto.

\section{Como citar el artículo:}

Agüero Pérez, M. M.; López Fraile, L. A. y Pérez Expósito J. (2019). Challenge Based Learning como modelo de aprendizaje profesionalizante. Caso del programa Universidad Europea con Comunica $+\mathrm{A}$. [Challenge Based Learning as a professional learning model. Universidad Europea and Comunica +A program case study]. Vivat Academia. Revista de Comunicación, (149), 1-24.

doi: http://doi.org/10.15178/va.2019.149.1-24

Recuperado de http://www.vivatacademia.net/index.php/vivat/article/view/1158

\section{INTRODUCCIÓN}

El acceso a enormes cantidades de información, la aplicación de las nuevas tecnologías o el auge de las dinámicas colaborativas hacen necesario una continua adaptación de las técnicas y actividades de aprendizaje que permitan esa adaptación a este escenario cambiante y a sus consecuencias en el mercado laboral, combinando conocimiento académico y aplicación real (Escamilla, 2008; Perrenoud, 2004 y 2012; Zabala y Arnau, 2007). Trabajar con proyectos reales mejora la motivación y eso redunda en un mejor aprendizaje de los estudiantes (Terron, Blanco y Velasco, 2017), a la vez, llevan a desenvolverse en un ambiente profesional acercándoles a sus futuras profesiones (Gallego, Terrón, Lagioia, y Valleni, 2015).

Estos nuevos paradigmas educativos desplazan el centro del tradicional sistema basado en el profesor a nuevos sistemas centrados en el alumno, creando así, alumnos

Vivat Academia. Revista de Comunicación. 15 diciembre 2019 /15 marzo 2020, nº 149, 1-24 
Agüero Pérez, M. M.; López Fraile, L. A. y Pérez Expósito J. Challenge Based Learning como modelo de aprendizaje profesionalizante. Caso del programa Universidad Europea con Comunica $+A$.

más participativos, comprometidos y una formación más personalizada (Zavirov, 2013), sintiendo su trabajo más importante y con más sentido de responsabilidad (Velasco, Gómez de Merodio y Terrón, 2017).

En esta línea, surge un nuevo método de aprendizaje basado en retos o desafíos: el Challenge Based Learning (CBL), basado en el uso de problemas como foco de la actividad educativa (Boud y Feletti, 2013). Es un método escalable y aplicable con pocas necesidades de cambio y basado en una idea: hacer el aprendizaje relevante (Johnson y Brown, 2011).

CBL es un nuevo marco educativo basado en el aprendizaje experiencial creado por la compañía americana Apple en el año 2008 y en el que se trata de generar soluciones a problemas reales de índole local o global, mientras que se construye una dinámica de aprendizaje donde se difuminan los límites entre alumno y profesor, empoderando a todos en un proceso de adquisición de habilidades y conocimientos, animando a la formación entre pares para desarrollar acciones reales que resuelven verdaderos problemas (Cheung, Cohen, Lo y Elia, 2011). El profesor actúa como facilitador del proceso en lugar de como proveedor de conocimiento (Hmelo-Silver, 2004).

Este proceso de participación activa en la formación es una herramienta muy efectiva para involucrar y motivar a estudiantes nacidos en la era digital y ayudarles a gestionar un método de resolución de problemas actuales reales a través del pensamiento crítico (Przybysz-Zaremba, Rimkūnienè, Vasilienè-Vasiliauskienė y Butvilas, 2017). Por otro lado, mejora la integración y relación de conceptos (O’Mahony, 2011).

CBL propugna una discusión sobre la tradicional división entre la formación académica y la formación profesionalizante a la vez que se propone como objetivo una estable colaboración entre ambos (Schmidt \& Gibbs, 2009) y en el mismo sentido obliga a una reorientación de las relaciones entre todos los actores (organizaciones educativas, empleadores, estudiantes y legisladores) implicados en la educación superior del futuro (McWilliam \& Taylor, 2001).

\subsection{Estructura básica del CBL}

CBL se basa en varios elementos clave que lo definen e identifican:

No existe diferencia entre alumno y profesor: todos los participantes son, a la vez, formadores y alumnos; todos aprenden y todos comparten la responsabilidad y el trabajo en la formación; el profesor es un colaborador en el aprendizaje y los estudiantes cuentan con la participación de expertos que les ayudan a mejorar su capacidad profesional.

Vivat Academia. Revista de Comunicación. 15 diciembre 2019 /15 marzo 2020, nº 149, 1-24 
Agüero Pérez, M. M.; López Fraile, L. A. y Pérez Expósito J. Challenge Based Learning como modelo de aprendizaje profesionalizante. Caso del programa Universidad Europea con Comunica $+A$.

La formación no está limitada a las aulas: se integran a otros actores como empleadores, asociaciones $\mathrm{u}$ otros sectores de la sociedad.

Son los alumnos quienes eligen e inspiran los contenidos de la formación, haciendo así mucho más integrador y motivante el proceso. Para ello, seleccionan un desafío o reto (challenge) que consideran relevante y apasionante para ellos en ese momento.

Los alumnos adquieren las habilidades necesarias que se demandan por los empleadores. Se les otorga un espacio de pensamiento crítico y creativo. El objetivo no se limita únicamente al producto final sino a todo el proceso de formación y trabajo hasta llegar a ese resultado.

El uso de la tecnología durante todo el proceso es crítico y permite generar una nueva experiencia de aprendizaje.

\subsection{Fases del proceso CBL}

El proceso de aprendizaje, en resumen, incluye: dar las «big ideas», desarrollar las cuestiones esenciales, los retos, actividades y cuestiones guía, soluciones y publicación del proceso (Johnson \& Adams, 2011). El proceso detallado se enumera a continuación:

Fase 1: Involucración

Idea General o «Big Idea»: concepto amplio y relevante para los alumnos y para la sociedad en general.

Pregunta Esencial o «Essential Question»: permite contextualizar y hacer más personal y operativa la Idea General.

Reto o «Challenge»: es una respuesta concreta a la Pregunta Esencial. Debe constituir una solución real y aplicable al problema detectado.

Fase 2: Investigación

Cuestiones Guía o «Guiding Questions»: son generadas por los estudiantes y ayudan a categorizar y priorizar, organizando así el proceso de aprendizaje.

Actividades Guía o «Guiding Activities»: métodos y herramientas que permiten responder a las cuestiones guía.

Análisis de lo aprendido en la definición y respuesta a las cuestiones planteadas.

Vivat Academia. Revista de Comunicación. 15 diciembre 2019 /15 marzo 2020, nº 149, 1-24 
Agüero Pérez, M. M.; López Fraile, L. A. y Pérez Expósito J. Challenge Based Learning como modelo de aprendizaje profesionalizante. Caso del programa Universidad Europea con Comunica $+A$.

Fase 3: Actuación

Solución: propuesta de solución planteada de forma argumentada, estructurada y razonada. Pueden existir infinitas soluciones para un mismo problema.

Implementación: puesta en marcha de la solución en una situación y ante un público reales.

Evaluación: medición de la efectividad de la solución propuesta y del proceso llevado a cabo.

Todo el proceso genera una serie de evidencias que sirven para la evaluación de la actividad, así como para la creación de un porfolio por parte del estudiante. Es importante la documentación de todo el proceso para permitir generar información que permita el análisis y la reflexión posterior con los estudiantes, haciendo así que el proceso sea realmente rico y relevante para ellos.

\subsection{Ventajas del CBL}

Incrementa la motivación y ayuda a la consecución de las metas educativas de los estudiantes (Baloian, Breuer, Hoeksema, Hoppe \& Milrad, 2004) y, así mismo, mejora los resultados generales de los estudiantes (Luis y Marrero, 2013).

Favorece la integración y adaptación de alumnos al mundo universitario desde el inicio, evitando la exclusión (Cancino, 2018).

Ayuda al alumnado en la adquisición de un aprendizaje auto dirigido (Savery, 2006).

Es un sistema abierto que permite que la responsabilidad de la formación y del aprendizaje se comparta entre el profesor y el alumno y en el que, además, participan expertos profesionales que contribuyen a la formación integral del alumno.

Construye una verdadera relación entre el mundo académico y el mundo profesional, propiciando una mayor capacidad de los estudiantes para la resolución de problemas (Finkelstein et al., 2010), generando así un impacto inmediato en la sociedad.

Genera un uso apropiado, correcto y concreto de las nuevas tecnologías como herramientas colaborativas (Premsmith, Wannapiroon y Nilsook, 2016) durante todo el proceso, para la investigación, información, presentación, análisis y comunicación de las propuestas planteadas. Además, desarrolla una interacción entre competencias técnicas y desarrollo emocional (Trujillo; Hinojo; Marín; Romero y Campos, 2015).

Vivat Academia. Revista de Comunicación. 15 diciembre 2019 /15 marzo 2020, nº 149, 1-24 
Agüero Pérez, M. M.; López Fraile, L. A. y Pérez Expósito J. Challenge Based Learning como modelo de aprendizaje profesionalizante. Caso del programa Universidad Europea con Comunica $+A$.

\subsection{Casos relevantes en otras instituciones educativas}

El uso de esta metodología ha sido demostrado como adecuada en numerosos casos de investigación dentro del sistema educativo de enseñanza superior, como son en el área de medicina (Carrión et al., 2015), ingeniería (de los Ríos et al, 2015; Morales et al., 2015), e incluso para la formación de docentes universitarios (Benítez et al., 2013). También en el ámbito internacional existen casos en Estados Unidos (Rice y Shannon, 2015) o en China (Xu y Liu, 2010).

Por ejemplo, podemos citar el caso del MIT, con su programa denominado Ideas Global Challenge. Este otorga un espacio para encontrar soluciones a problemas globales. Su estructura permite desarrollar prototipos y propuestas concretas y la implantación de dichas soluciones durante el lapso temporal de un año. Los estudiantes reciben formación en innovación, gestión de proyectos de ingeniería, en emprendimiento y en incubadoras de negocio. El equipo ganador de esta competición anual recibe un premio de 15.000 dólares (MIT, 2015).

Otro caso lo encontramos en la Aalborg University. Esta institución ofrece un programa internacional llamado Problem Based Learning Model. En este programa el estudiante tiene la oportunidad de trabajar durante cuatro semestres en empresas externas, enfrentándose durante este tiempo a problemas reales, lo que les permite desarrollar habilidades para dar soluciones creativas a situaciones profesionales cotidianas (Aalborg University, s. f.).

Siguiendo con casos de referencia relevantes, en la Montana State University tienen un programa cuyo objetivo es el de evaluar el efecto del sistema challenge based learning en la motivación y en los resultados de los estudiantes de primer año de Ciencias Físicas, quienes durante el desarrollo del programa deben diseñar e implementar un proyecto medio ambiental que beneficie a la sociedad. Las propuestas se presentan en formato audiovisual. La implementación dura seis semanas durante las que se responde a las cuestiones planteadas y se plantean soluciones al reto. Los resultados indican que esta estrategia incrementa la motivación de los estudiantes y mantiene los niveles de consecución de objetivos incluso con cargas de trabajo superiores en un 50\% a los alumnos que no siguieron el programa. El 53\% de los estudiantes creen que el conocimiento adquirido con este reto es importante para su futuro profesional (Swiden, 2013).

En la Universidad de Connecticut, su Experiential Learning Collaborative ofrece a los estudiantes la oportunidad de adquirir habilidades de liderazgo y gestión de equipos, mientras desarrollan proyectos para empresas, instituciones y organizaciones sin ánimo de lucro. Los estudiantes asimilan y aplican habilidades directivas a situaciones de negocios reales y hacen networking con los líderes de la industria y del sector en el que

Vivat Academia. Revista de Comunicación. 15 diciembre 2019 /15 marzo 2020, nº 149, 1-24 
Agüero Pérez, M. M.; López Fraile, L. A. y Pérez Expósito J. Challenge Based Learning como modelo de aprendizaje profesionalizante.

Caso del programa Universidad Europea con Comunica $+A$.

trabajan. Los proyectos implican una estrecha colaboración entre el cliente y los equipos de estudiantes que son guiados por profesores y/o mentores con experiencia en la casuística del sector profesional relacionado. Uno de los partners relacionados con el programa fue la empresa Henkel, cuyo directivo Brad Wade indicó al finalizar las presentaciones finales que le sorprendió el nivel de preparación y profesionalidad que reflejaban los estudiantes que participaron en el proyecto y que habían recibido la formación experiencial based learning. Por otro lado, una de las estudiantes participantes manifestó que "el programa realmente crea confianza sabiendo que puedes analizar un negocio, descubrir problemas, proporcionar soluciones y presentar todo esto ante un cliente" (Hall, 2018).

Otro de los casos de referencia es el de Universidad de Harvard y su President's Challenge. Este programa se desarrolla a través del Harvard Innovation Lab, y su finalidad es la de ayudar a los estudiantes a formular soluciones a problemas complejos a través de la confrontación y examen de importantes cuestiones de la actualidad. El programa se divide en dos fases: la primera, consistente en la presentación de las propuestas por parte de los equipos; en una segunda fase, el jurado emite un veredicto de los proyectos que seguirán adelante, lo que desemboca en que cada equipo recibe financiación para desarrollar su propuesta. El equipo ganador recibe un premio final de 410.000 dólares. El proyecto está enfocado en áreas específicas como son la innovación educativa, soluciones para temas de salud, energía y medioambiente, desarrollo económico, empleo sostenible y ciudades conectadas (Harvard Innovation Lab, 2015).

Para finalizar este repaso por algunos casos de éxito de implantación de CBL, podemos citar la puesta en marcha que hacen en el Tecnológico de Monterrey. En esta universidad llevan a cabo diversos proyectos basados en CBL con cifras sorprendentes de participación: un total de 50.000 estudiantes, más de 3.000 profesores y con más de 1.800 proyectos involucrados desarrollados con CBL. Cada challenge se llevó a cabo por grupos compuestos por 15 estudiantes. Los alumnos participantes en los retos valoraron positivamente los siguientes ítems de la encuesta de satisfacción que cumplimentaban al finalizarlos: interacción, capacidad de afrontar retos de la vida real, contacto profesional, innovación y aplicación de conceptos. Uno de los más relevantes es el denominado "Rediseño de la red de distribución del helado Holanda en México". El reto consiste en diagnosticar la situación presente de la empresa y proponer vías de mejora del sistema de distribución. Tras resolver el reto, las competencias más desarrolladas y que se convirtieron, por tanto, en claros beneficios del programa, percibidos por los estudiantes, fueron: un conocimiento más profundo sobre el tema, el desarrollo del pensamiento crítico, la capacidad de liderazgo, la tolerancia a la frustración y la comunicación oral y escrita (Membrillo-Hernández et al., 2018).

Vivat Academia. Revista de Comunicación. 15 diciembre 2019 /15 marzo 2020, nº 149, 1-24 
Agüero Pérez, M. M.; López Fraile, L. A. y Pérez Expósito J. Challenge Based Learning como modelo de aprendizaje profesionalizante. Caso del programa Universidad Europea con Comunica $+A$.

\subsection{CBL como metodología educativa aplicada}

Por todo lo citado con anterioridad, la facultad de Ciencias Sociales y de la Comunicación de la Universidad Europea de Madrid (UEM), en el curso académico 2016/2017, elige este modelo por ser un paradigma activo que cumple con las necesidades de aprendizaje de los estudiantes de las cuatro áreas de la facultad (empresa, educación, jurídica y comunicación) ya que es una metodología que trae la empresa al aula y que lleva el aula a la empresa. Además, es la metodología que mejor responde a las exigencias de las agencias acreditadoras en España.

A su vez, la agencia de publicidad española Comunica $+\mathrm{A}(\mathrm{C}+\mathrm{A})$ inmersa en un proceso de transformación cultural y con el objetivo de ganar notoriedad y captar talento, firma un convenio de colaboración con la Universidad Europea para poner en marcha un proyecto novedoso en la formación universitaria española. El proyecto consiste en la creación de un programa de formación dual, relacionando la empresa y la universidad como centros de formación experiencial y aproximación a la realidad profesional basado en CBL.

\section{OBJETIVOS}

El propósito fundamental del proyecto es conseguir una mayor integración con el mundo profesional de los alumnos del área de publicidad, que entronca con el objetivo estratégico de la facultad (el aula en la empresa y la empresa en el aula) y con el de propia agencia (captación de talento), para que los estudiantes puedan satisfacer las necesidades reales del mercado laboral, reduciendo así la distancia entre las actividades docentes y los retos profesionales en un mismo entorno de aprendizaje. Las premisas sobre las que se construye el programa son: recibir formación de los responsables de departamento de la agencia en sus propias oficinas conforme a un programa de formación previamente establecido por la UEM, trabajar con briefings reales de los clientes de la agencia y obtener una doble evaluación (feedback) por parte de profesores del área de conocimiento y de profesionales de la agencia.

Ante este entorno de aprendizaje planteado, el presente estudio indaga sobre la siguiente hipótesis: la utilización de la metodología CBL ayuda a mejorar los aspectos académicos, competenciales y de empleabilidad de los futuros egresados en Publicidad. De ella, se desprenden los siguientes objetivos:

Objetivo 1: verificar que el programa acerca a los estudiantes a la realidad profesional.

Objetivo 2: comprobar que los alumnos pueden cumplir con las exigencias profesionales de un cliente real.

Vivat Academia. Revista de Comunicación. 15 diciembre 2019 /15 marzo 2020, nº 149, 1-24 
Agüero Pérez, M. M.; López Fraile, L. A. y Pérez Expósito J. Challenge Based Learning como modelo de aprendizaje profesionalizante. Caso del programa Universidad Europea con Comunica $+A$.

Objetivo 3: comprobar que el programa facilita la integración de la formación teórica y práctica dentro de un ambiente profesional.

\section{METODOLOGÍA}

La metodología que se ha desarrollado para poner en marcha este programa se ha enmarcado en el modelo académico CBL, implantado en la Facultad de Ciencias Sociales y de la Comunicación de la UEM, ya que es la metodología que prepara a los estudiantes para que afronten retos reales y que refleja ámbito laboral del siglo XXI (digitalpromise.org, 2017). El proyecto CBL realizado por el departamento de Comunicación de la Universidad Europea de Madrid y la agencia de publicidad Comunica + A se ha realizado, hasta el momento, en dos ediciones: una primera edición en el curso 16/17 y una segunda en el curso 17/18. El programa, en las dos ediciones, tiene las mismas premisas (tal y como se han expuesto en el epígrafe 1): recibir formación de los responsables de departamento de la agencia en sus propias oficinas conforme a un programa de contenidos que previamente se ha establecido por los responsables del departamento de comunicación de la universidad, trabajar con briefings reales de los clientes de la agencia y obtener una doble evaluación por parte de profesores del área de conocimiento y de profesionales de la agencia. Por tanto, y siguiendo la metodología CBL, la big idea o idea general sería la profesionalización de los estudiantes, la essential question sería ¿cómo resuelven los estudiantes un problema de comunicación de un anunciante?, el challenge o reto sería que los estudiantes den una respuesta en forma de campaña a dicho problema comunicativo planteado por la agencia de la mano de un anunciante, y el assessment o evaluación (paso fundamental en la metodología CBL) que sería doble ya que los estudiantes reciben la evaluación de los profesores y también de los profesionales de la agencia, siendo esta última la que hace culminar el programa con una recompensa tangible en forma de unas prácticas universitarias remuneradas en la agencia para los estudiantes mejor calificados. Del análisis de los resultados de la primera fase se realizan una serie de mejoras que se incorporaron en la segunda fase, en un proceso de mejora continua cuyo objetivo es maximizar el desarrollo de las competencias profesionalizantes.

Primera edición (curso 16/17): en octubre de 2016, el departamento de comunicación de la UE y la agencia de publicidad C+A diseñan el programa, que dará respuesta a los objetivos planteados en el epígrafe 1, y se plantea en las siguientes fases:

Fase 1. Información y selección de candidatos.

1. Charla de presentación y motivación a los estudiantes del área de publicidad de la universidad.

2. Selección de los candidatos conforme a unos criterios pactados con la agencia (propuesta de viralización del propio programa). Se hace una selección de 14

Vivat Academia. Revista de Comunicación. 15 diciembre 2019 /15 marzo 2020, nº 149, 1-24 
Agüero Pérez, M. M.; López Fraile, L. A. y Pérez Expósito J. Challenge Based Learning como modelo de aprendizaje profesionalizante. Caso del programa Universidad Europea con Comunica $+A$.

participantes, de un total de 25 candidaturas. Los 14 participantes se organizan en duplas en función de sus relaciones y/o afinidades.

Fase 2. Desarrollo del programa.

1. El programa está compuesto por seis charlas formativas (un martes cada 15 días, desde las 13:00 a las 16:30 horas) con un tema central (retail marketing, branded content, acciones BTL, servicios al cliente, publicidad digital, presupuestos y ROI) lideradas por los directores de los departamentos de la agencia, más un lanzamiento de briefing real de un cliente.

2. Los estudiantes preparan durante una semana una solución estratégica y creativa que dé respuesta al briefing planteado por la agencia-cliente (es en esta fase donde los estudiantes generan sus guiding questions o preguntas guías que organizan su proceso de aprendizaje).

3. Los estudiantes tienen una sesión de debrief con tres profesores del departamento (especializados en el contenido que los alumnos han recibido en la sesión de la agencia). Los profesores asesoran, dan pautas de corrección y presentación para que los estudiantes lo incorporen en la presentación que realizarán en la agencia (esta fase sería, en la metodología CBL, the guiding activities o actividades guía).

4. Los estudiantes acuden a la sesión posterior que está divida en tres partes (siguiente sesión de contenido, más presentación por parte de los estudiantes de la solución al briefing planteado en la formación anterior y, por último, feedback del trabajo realizado).

Fase 3. Finalización del programa. Tras la última sesión de contenido y resolución de briefing, se produce una reunión entre los responsables del programa en la agencia y los responsables del programa en la universidad y eligen a los cuatro mejores estudiantes, que realizarán unas prácticas remuneradas en la agencia (esta fase corresponde con la fase de actuación de la metodología CBL).

Tras la primera edición, se pasa un cuestionario a los estudiantes participantes, con un total de 12 preguntas con respuestas cerradas, y una valoración del 1 al 5, siendo 1 el valor más bajo y 5 el más alto. Las preguntas se diseñan conforme a las siguientes áreas: área académica (integración con los contenidos formativos del grado), área competencial (competencias transversales), área de empleabilidad (cercanía del programa a la profesión-competitividad profesional en el mercado); además, se deja un espacio para que los estudiantes puedan detallar 3 puntos fuertes y 3 áreas de mejora y, por último, se abre un ítem adicional para que el estudiante pueda valorar del 1 al 10 su satisfacción general con el programa.

Una vez analizados los cuestionarios de los estudiantes, los comentarios de los ponentes-directores de los departamento de la agencia y los comentarios de los profesores, los responsables del programa, tanto por la agencia como por la universidad,

Vivat Academia. Revista de Comunicación. 15 diciembre 2019 /15 marzo 2020, nº 149, 1-24 
Agüero Pérez, M. M.; López Fraile, L. A. y Pérez Expósito J. Challenge Based Learning como modelo de aprendizaje profesionalizante. Caso del programa Universidad Europea con Comunica $+A$.

se sientan a diseñar la siguiente edición $(17 / 18)$ y se incluyen los siguientes cambios: se nombrará un tutor profesional de la agencia por cada uno de los grupos participantes para mejorar el feedback y el seguimiento, se abre la participación a estudiantes de la titulación de marketing para que el proyecto gane en transversalidad de conocimientos, la elección de los grupos será dirigida (se mezclarán estudiantes de la rama de creatividad, estrategia, marketing y de distintos cursos), se lanzará un único brief real al comienzo del programa y se trabajará sobre él en todas las sesiones, las charlas estarán compuestas por la sesión de formación más una reunión de tutoría. Al final del programa, todos los grupos presentarán su propuesta final frente a un tribunal compuesto por la representante de la marca sobre la que trabajan los alumnos y los profesionales de $\mathrm{C}+\mathrm{A}$ y dos profesores de la universidad. Por lo tanto, el programa queda de la siguiente manera:

Fase 1. Información y selección de candidatos.

1. Charla de presentación y motivación a los estudiantes del área de publicidad y de marketing de la universidad.

2. Selección de los candidatos conforme a unos criterios pactados con $\mathrm{C}+\mathrm{A}$ (propuesta de viralización del propio programa). Se hace una selección de 16 participantes, de un total de 30 candidaturas. La organización de los grupos (de 4 componentes cada uno) se hace bajo los criterios especificados en el párrafo anterior.

Fase 2. Desarrollo del programa.

1. El programa está compuesto por seis charlas formativas (un martes cada 15 días, desde las 13:00 a las 16:30 horas) con un tema central (cuentas \& planning, creación, digital, retail marketing, BTL, presupuestos y ROI) lideradas por los directores de los departamentos de la agencia. Durante la primera sesión en C+A, se lanza el briefing sobre el que trabajarán los alumnos durante todo el programa y cada grupo se pone en contacto con su tutor profesional.

2. Los estudiantes, durante los quince días que transcurren entre las sesiones, trabajan en los objetivos y soluciones planteados por el briefing y están en contacto con sus tutores profesionales (es en esta fase donde los estudiantes generan sus guiding questions o preguntas guías que organizan su proceso de aprendizaje). Dos días antes de la siguiente sesión, los alumnos deben mandar sus presentaciones a los tutores para que puedan revisarlas juntos de manera presencial.

3. Los estudiantes acuden a la sesión posterior que está divida en dos partes: a. siguiente sesión de contenido, b. reunión con los tutores para corrección de ideas y planteamientos (en este momento y según la metodología CBL, se realizarían the guiding activities o actividades guía).

Vivat Academia. Revista de Comunicación. 15 diciembre 2019 /15 marzo 2020, nº 149, 1-24 
Agüero Pérez, M. M.; López Fraile, L. A. y Pérez Expósito J. Challenge Based Learning como modelo de aprendizaje profesionalizante. Caso del programa Universidad Europea con Comunica $+A$.

Fase 3. Finalización del programa o fase de actuación en la metodología CBL. Quince días después de la última sesión de contenido, se desarrolla una sesión final en la que los cuatro grupos presentan su propuesta a los siguientes jurados: representante del cliente, representantes de la agencia y representantes de los profesores de la universidad. Los alumnos disponen de 20 minutos para plantear y desarrollar su idea/plan estratégico y de comunicación; al finalizar las presentaciones se produce una reunión entre los miembros del jurado $\mathrm{y}$, en función de una rúbrica previamente pactada, se da feedback a cada uno de los grupos y se elige el grupo que mejor haya dado respuesta al briefing y haya aportado la idea que más guste al cliente. Igual que en la edición anterior, y como resultado del programa, cada miembro del grupo ganador recibe unas prácticas remuneradas en la agencia.

\section{RESULTADOS}

El cuestionario que se pasó a los veintinueve estudiantes que formaron parte activa del proyecto (uno alumno de la segunda edición dejó el programa por cuestiones personales), se compone de tres áreas diferenciadas: el área académica, el área de competencias y el área de empleabilidad. Para cada una de estas áreas se formularon 4 preguntas específicas, cuyos resultados estadísticos se detallan a continuación.

En lo que concierne al área académica, en la figura 1 podemos observar una clara tendencia hacia la valoración "bastante", que equivale a una puntuación de 4 sobre 5 , es decir, un grado evaluativo alto por parte de los estudiantes. Hay que destacar que un $31 \%$ de los mismos indican que el programa les ha ayudado "mucho" y un 51,7\% "bastante" a mejorar sus presentaciones orales y escritas para las asignaturas del grado. Destacar también que el ítem relativo a la mejora de los conocimientos y comprensión de conceptos relacionados con el grado ha obtenido una calificación de "bastante" en un $72,4 \%$ de los casos. Como aspecto de mejora, destaca un ítem donde dicho programa no ha tenido el impacto deseado. Concretamente el $48,3 \%$ de los estudiantes señalan que el programa les ha ayudado a mejorar solamente "algo" sus calificaciones en las asignaturas del grado.

Finalmente decir que el área académica obtiene una media global de un 3,9, calificación que la sitúa en una valoración muy positiva. Esto nos permite concluir que el desarrollo académico adquiere un soporte importantísimo con la puesta en marcha de programas, como el analizado en la presente investigación, donde los alumnos complementan los contenidos académicos con la puesta en marcha de casos reales, que acrecientan el nivel de conocimientos y resto de objetivos académicos propios de la profesión publicitaria.

Vivat Academia. Revista de Comunicación. 15 diciembre 2019 /15 marzo 2020, nº 149, 1-24 
Agüero Pérez, M. M.; López Fraile, L. A. y Pérez Expósito J. Challenge Based Learning como modelo de aprendizaje profesionalizante.

Caso del programa Universidad Europea con Comunica $+A$.

Tabla 1. Análisis del área académica del cuestionario.

\begin{tabular}{|c|l|c|c|c|c|c|}
\hline & $\begin{array}{l}\text { EN QUÉ MEDIDA EL } \\
\text { PROGRAMA DE } \\
\text { COMUNICA+A TE } \\
\text { HA AYUDADO A } \\
\text { MEJORAR TU/TUS: }\end{array}$ & NADA & $\begin{array}{c}\text { MUY } \\
\text { POCO }\end{array}$ & ALGO & BASTANTE & MUCHO \\
\cline { 2 - 7 } & $\begin{array}{l}\text { Conocimiento y } \\
\text { comprensión de } \\
\text { conceptos } \\
\text { relacionados del } \\
\text { Grado }\end{array}$ & $0 \%$ & $0 \%$ & $10,3 \%$ & $72,4 \%$ & $17,2 \%$ \\
\cline { 2 - 7 } & $\begin{array}{l}\text { Calificaciones en las } \\
\text { asignaturas del Grado }\end{array}$ & $3,4 \%$ & $13,8 \%$ & $48,3 \%$ & $34,5 \%$ & $0 \%$ \\
\cline { 2 - 7 } & $\begin{array}{l}\text { Presentaciones orales } \\
\text { y escritas para las } \\
\text { asignaturas del Grado }\end{array}$ & $0 \%$ & $3,4 \%$ & $13,8 \%$ & $51,7 \%$ & $31,0 \%$ \\
\hline $\begin{array}{l}\text { Conocimientos } \\
\text { adquiridos en clase }\end{array}$ & $0 \%$ & $0 \%$ & $17,2 \%$ & $58,6 \%$ & $24,1 \%$ \\
\hline & \begin{tabular}{l} 
Media \\
\hline
\end{tabular}
\end{tabular}

Fuente: Elaboración propia a partir de datos de la investigación.

En lo atinente al área de competencias, podemos constatar (tabla 2) que la valoración «bastante» ha sido la mayoritariamente puntuada para todos los items incluidos, seguida muy de cerca por la de "mucho". Destacar que el 41,4\% puntuan con un 4 sobre 5 aspectos como la mejora que el programa ha supuesto para ellos en su capacidad para recibir críticas, realizar argumentaciones, cumplir con tareas y plazos, mejorar el clima de trabajo y en la organización y distribución de tareas.

El área de competencias obtiene una calificación global final de un 3,8, situándose, por tanto, en la parte alta de las valoraciones de los encuestados, lo que viene a reflejar que los estudiantes han incorporado a su bagaje experiencial, la inestimable importancia del desarrollo de competencias afines a la profesión, sin las cuales, la ubicación laboral sería difícil de conseguir.

Tabla 2. Análisis del área de competencias del cuestionario.

\begin{tabular}{|c|c|c|c|c|c|c|}
\hline 至 & $\begin{array}{l}\text { EN QUÉ MEDIDA EL } \\
\text { PROGRAMA DE } \\
\text { COMUNICA+A TE HA } \\
\text { AYUDADO A MEJORAR } \\
\text { TU CAPACIDAD: }\end{array}$ & NADA & $\begin{array}{l}\text { MUY } \\
\text { POCO }\end{array}$ & ALGO & BASTANTE & MUCHO \\
\hline 孚 & $\begin{array}{l}\text { Para hacer y recibir } \\
\text { críticas y tu capacidad de } \\
\text { argumentación }\end{array}$ & $0 \%$ & $13,8 \%$ & $34,5 \%$ & $41,4 \%$ & $10,3 \%$ \\
\hline
\end{tabular}

Vivat Academia. Revista de Comunicación. 15 diciembre 2019 /15 marzo 2020, nº 149, 1-24 
Agüero Pérez, M. M.; López Fraile, L. A. y Pérez Expósito J. Challenge Based Learning como modelo de aprendizaje profesionalizante.

Caso del programa Universidad Europea con Comunica $+A$.

\begin{tabular}{|l|l|l|l|l|l|}
\hline $\begin{array}{l}\text { De planificación de } \\
\text { objetivos, establecer } \\
\text { plazos, concretar y } \\
\text { ordenar prioridades y } \\
\text { organizar por objetivos y } \\
\text { evaluar tu evolución }\end{array}$ & $0 \%$ & $17,2 \%$ & $13,8 \%$ & $34,5 \%$ & $34,5 \%$ \\
\hline $\begin{array}{l}\text { De cumplir plazos y } \\
\text { tareas, tu implicación en } \\
\text { el trabajo y tu } \\
\text { orientación hacia la } \\
\text { calidad de los trabajos } \\
\text { presentados }\end{array}$ & $0 \%$ & $13,8 \%$ & $17,2 \%$ & $37,9 \%$ & $31,0 \%$ \\
\hline $\begin{array}{l}\text { En el cumplimiento de } \\
\text { tareas y plazos, mejorar } \\
\text { el clima de trabajo, } \\
\text { organización y } \\
\text { distribución de tareas }\end{array}$ & $3,4 \%$ & $13,8 \%$ & $13,8 \%$ & $41,4 \%$ & $27,6 \%$ \\
\hline Media & & 3,8 & \\
\hline
\end{tabular}

Fuente: Elaboración propia a partir de datos de la investigación.

Respecto al área de empleabilidad (tabla 3), también la puntuación mayoritariamente obtenida en todos los items ha sido la del 4 ("bastante"). Destacamos como punto altamente relevante que los encuestados han puntuado "mucho", en un 55,2\% de los casos, el item que medía la utilidad de los conocimientos obtenidos a través del programa a la hora llevarlos a la práctica profesional, según lo demandado por el mercado. Como punto de mejora se manifiesta el item vinculado a la utilidad que tendrá el networking (red de contactos) conseguido en el programa en la futura carrera profesional de los estudiantes que, aunque obtiene un $44,8 \%$ de "bastantes", también es valorado solamente con un "algo" en el $31 \%$ de las ocasiones.

El área de empleabilidad es el que obtiene una mejor valoración global de las tres áreas analizadas, con una puntuación global final de 4 sobre 5 . Teniendo en cuenta que el factor de la empleabilidad es, tal vez, el más relevante a la hora de que los estudiantes obtengan una utilidad palpable del programa en su futuro profesional, es muy valorable que su calificacion final haya arrojado un resultado tan alto.

Tabla 3. Análisis del área de empleabilidad del cuestionario.

\begin{tabular}{|c|c|c|c|c|c|c|}
\hline \multirow{2}{*}{ 空 } & $\begin{array}{l}\text { EN QUÉ MEDIDA EL } \\
\text { PROGRAMA DE } \\
\text { COMUNICA+A: }\end{array}$ & NADA & $\begin{array}{l}\text { MUY } \\
\text { POCO }\end{array}$ & ALGO & BASTANTE & MUCHO \\
\hline & $\begin{array}{l}\text { Te ha ayudado a mejorar tu } \\
\text { capacidad de realizar y } \\
\text { pasar con éxito entrevistas } \\
\text { personales de selección }\end{array}$ & $10,3 \%$ & $6,9 \%$ & $27,6 \%$ & $41,4 \%$ & $13,8 \%$ \\
\hline
\end{tabular}

Vivat Academia. Revista de Comunicación. 15 diciembre 2019 /15 marzo 2020, nº 149, 1-24 
Agüero Pérez, M. M.; López Fraile, L. A. y Pérez Expósito J.

Challenge Based Learning como modelo de aprendizaje profesionalizante.

Caso del programa Universidad Europea con Comunica $+A$.

\begin{tabular}{|l|l|c|c|c|c|}
\hline $\begin{array}{l}\text { Te otorga mayor facilidad } \\
\text { de ser contratado que antes } \\
\text { de haberlo realizado }\end{array}$ & $0 \%$ & $10,3 \%$ & $13,8 \%$ & $41,4 \%$ & $34,5 \%$ \\
\hline $\begin{array}{l}\text { En qué medida crees que los } \\
\text { conocimientos obtenidos en } \\
\text { el programa de } \\
\text { COMUNICA+A son útiles } \\
\text { en la práctica profesional } \\
\text { demandada por el mercado }\end{array}$ & $0 \%$ & $0 \%$ & $6,9 \%$ & $37,9 \%$ & $55,2 \%$ \\
\hline $\begin{array}{l}\text { Crees que los contactos } \\
\text { (networking) hechos en el } \\
\text { programa de } \\
\text { COMUNICA+A te pueden } \\
\text { ayudar en tu futura carrera } \\
\text { profesional }\end{array}$ & $3,4 \%$ & $10,3 \%$ & $31,0 \%$ & $44,8 \%$ & $10,3 \%$ \\
\hline Media & & 4 & \\
\hline
\end{tabular}

Fuente: Elaboración propia a partir de datos de la investigación.

Finalmente, nuestro estudio estadístico finalizó con la evaluación de la satisfacción global por parte de los participantes, que han arrojado una media de 8 sobre 10, teniendo además en cuenta que el valor 8 obtuvo un 48,3\% de las respuestas. Este dato supone obtener un resultado muy positivo del programa que, acompañado por el análisis cualitativo que se realizó, ha permitido evaluar no sólo las bondades del programa sino también sus áreas de mejora.

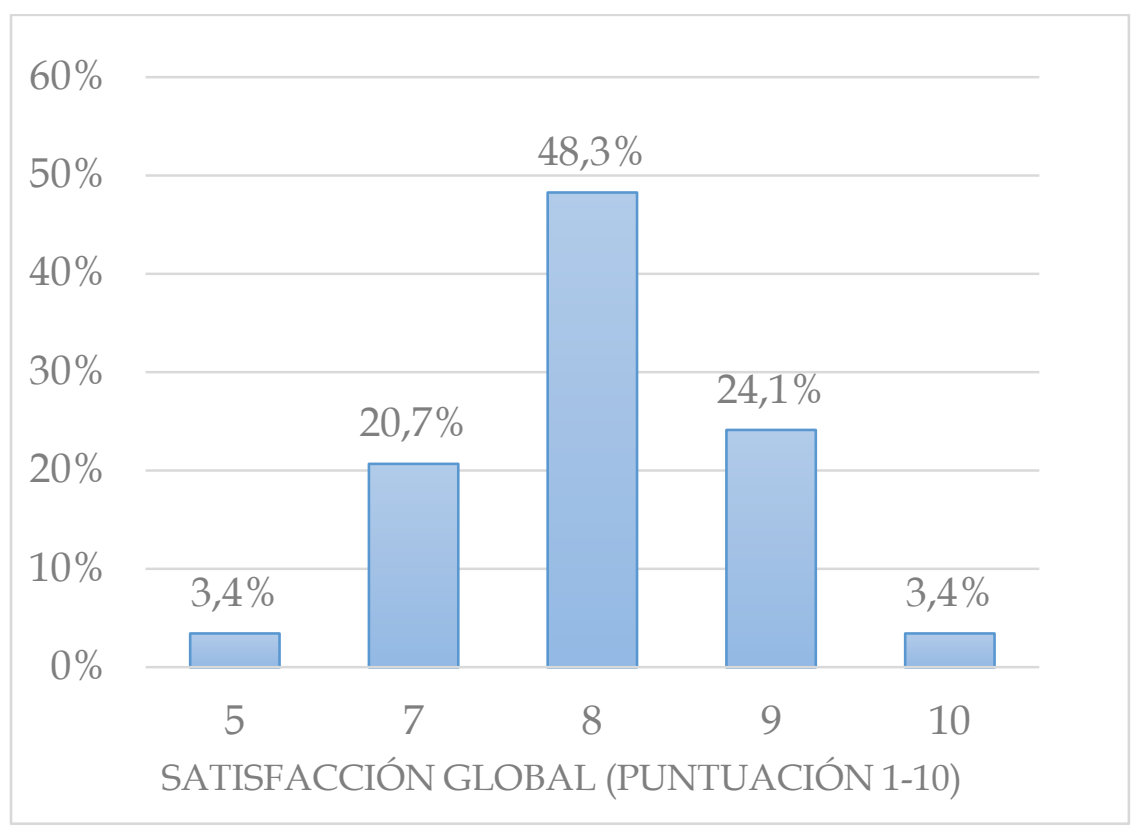

Gráfico 1. Satisfacción global con el programa.

Fuente: Elaboración propia a partir de datos de la investigación.

Vivat Academia. Revista de Comunicación. 15 diciembre 2019 /15 marzo 2020, nº 149, 1-24 
Agüero Pérez, M. M.; López Fraile, L. A. y Pérez Expósito J. Challenge Based Learning como modelo de aprendizaje profesionalizante.

Caso del programa Universidad Europea con Comunica $+A$.

Dentro de los cuestionarios cumplimentados por los alumnos participantes, se les solicitaba que aportaran tres aspectos positivos que, en su opinión, destacasen del programa. Una vez analizados los comentarios, en el apartado de «aspectos positivos» de la encuesta se obtienen los siguientes resultados:

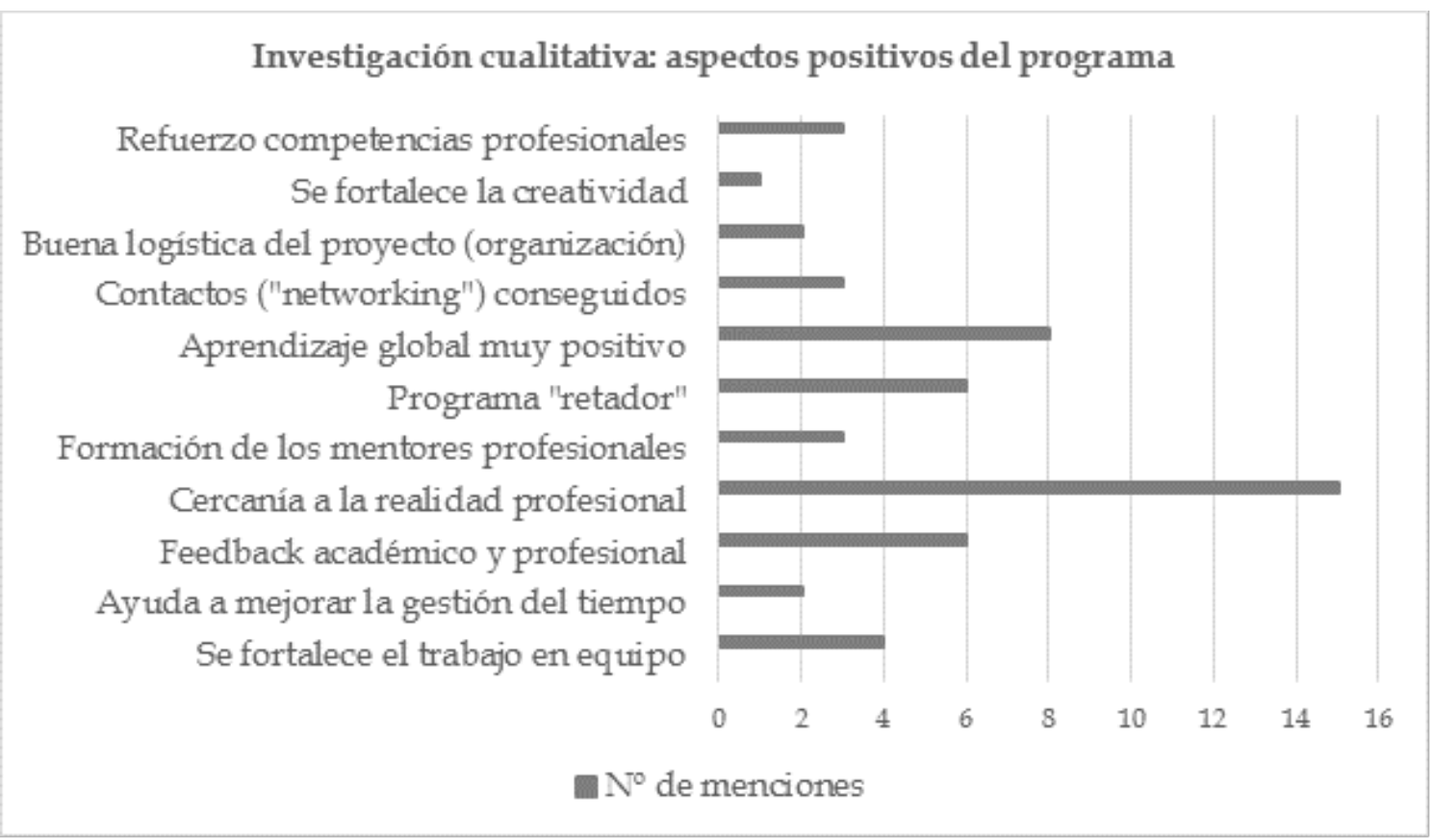

Gráfico 2. Numero de menciones en el campo de aspectos positivos del programa.

Fuente: Elaboración propia a partir de datos de la investigación.

El resultado más repetido con gran diferencia sobre el resto de las opiniones es la "cercanía a la realidad" (15 menciones), mientras que en segundo lugar aparece "aprendizaje global muy positivo" (8 menciones) y en tercer lugar, con el mismo número de menciones, los ítems "programa retador" y "feedback académico y profesional" (6 menciones cada uno).

En un segundo bloque, aparecen el resto de los ítems con un número de menciones menor (entre 1 y 4 menciones): "trabajo en equipo" (4 menciones), "refuerzo de competencias profesionales" (3 menciones), "networking" (3 menciones), "mejora de la gestión del tiempo"» (2 menciones) y "fortalece la creatividad" (1 mención).

También cabe comentar que la buena organización del programa obtiene 2 menciones.

Como aspectos de mejora señalados por los estudiantes en los cuestionarios destacamos las 5 repeticiones del ítem "mejora de las vías de comunicación con los mentores" y del ítem "no debe orientarse a cursos inferiores"; en un segundo lugar

Vivat Academia. Revista de Comunicación. 15 diciembre 2019 /15 marzo 2020, nº 149, 1-24 
Agüero Pérez, M. M.; López Fraile, L. A. y Pérez Expósito J. Challenge Based Learning como modelo de aprendizaje profesionalizante.

Caso del programa Universidad Europea con Comunica $+A$.

aparece el ítem "evitar solapamientos con el horario de clase" con tres repeticiones mientras que con dos repeticiones encontramos los ítems "mejora de la visibilidad del programa", "refuerzo del conocimiento de todas las áreas de la agencia", "equilibrio del nivel de conocimientos de los grupos" y "refuerzo conocimientos para cursos inferiores".

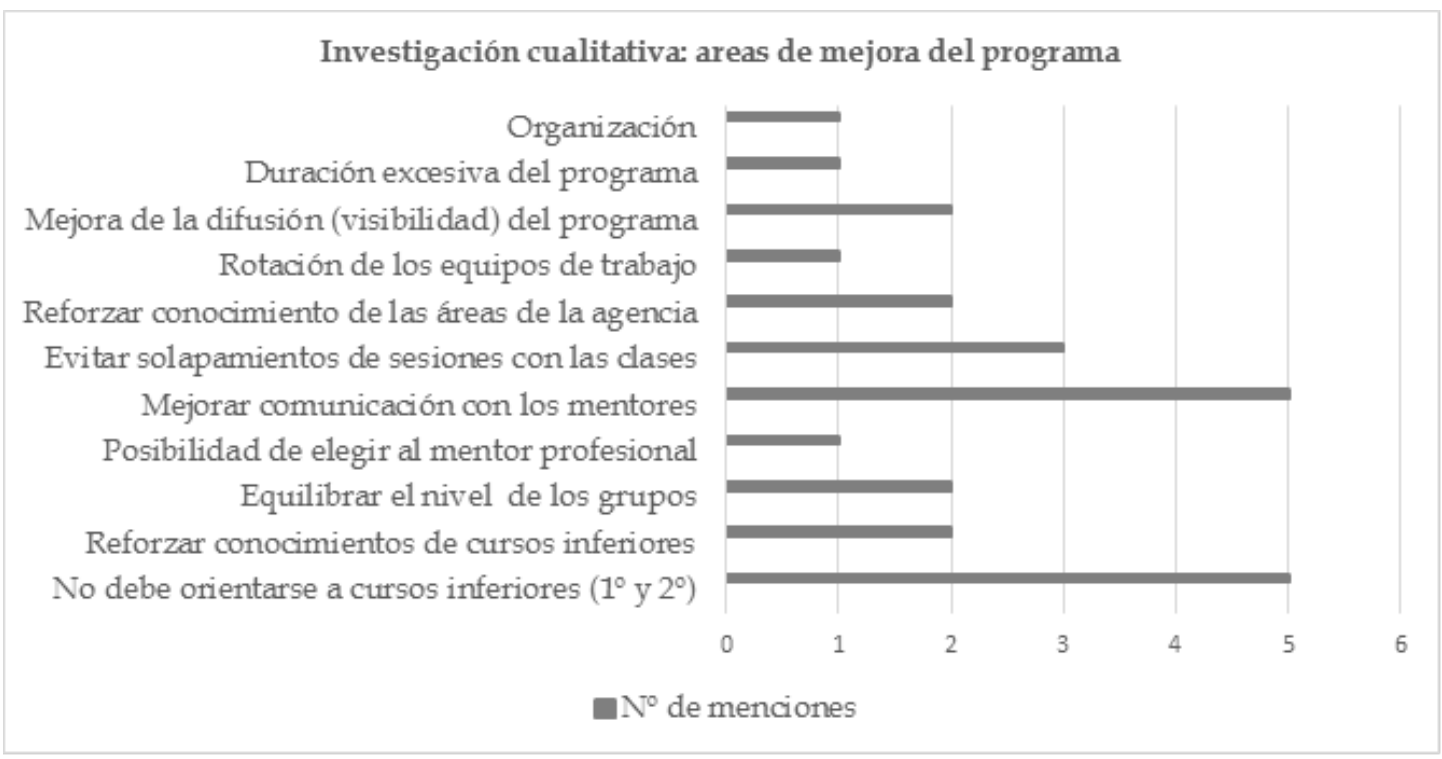

Gráfico 3. Numero de menciones en el campo de aspectos a mejorar del programa. Fuente: Elaboración propia a partir de datos de la investigación.

\section{DISCUSIÓN}

La empleabilidad y la cercanía a las profesiones (tal y como se ha revisado en el apartado introductorio) es uno de los objetivos del plan estratégico de la Facultad de Ciencias Sociales y de la Comunicación de la UEM y, por tanto, es una prioridad para sus profesores, que están convencidos de que su compromiso con los estudiantes pasa por formarles para un mundo real, en un entorno competitivo en el que deberán ser capaces de liderar la transformación sociocultural y digital.

El departamento de comunicación de la universidad acepta el reto de utilizar la metodología CBL con los estudiantes del grado en publicidad gracias a la ayuda de la agencia española de publicidad $\mathrm{C}+\mathrm{A}$, convencidos de que puede ayudar a los estudiantes en la obtención de sus conocimientos académicos, sus áreas competenciales $y$, por supuesto, en su empleabilidad futura.

Una vez analizados los resultados de la investigación realizada, podemos afirmar sin duda que el CBL ayuda en el acercamiento del mundo profesional al académico y mejora la empleabilidad de los alumnos que participan en el programa, tal y como se

Vivat Academia. Revista de Comunicación. 15 diciembre 2019 /15 marzo 2020, nº 149, 1-24 
Agüero Pérez, M. M.; López Fraile, L. A. y Pérez Expósito J. Challenge Based Learning como modelo de aprendizaje profesionalizante.

Caso del programa Universidad Europea con Comunica $+A$.

había planteado en el objetivo 3, y atestiguan los estudiantes con la calificación más alta del programa (4 sobre 5) y con el mayor número de repeticiones en la valoración cualitativa del programa (15) en la afirmación "cercanía a la realidad profesional". Así mismo, podemos observar que, en los aspectos competenciales, el programa realizado bajo la metodología CBL, ayuda a los alumnos a recibir críticas y al cumplimiento de las tareas en los tiempos estimados, competencias que verán demandadas en el mundo laboral, por lo que podemos inferir que se cumple también el objetivo 2 planteado en esta investigación. Por otro lado, dentro del área académica, la mayoría de los estudiantes $(72,4 \%)$ valora muy positivamente el refuerzo que el programa hace de los conocimientos propios de su futura profesión y, con esta, afirmación, daríamos por cumplido el objetivo 3 de la investigación.

Sin duda, uno de los aspectos que ratifica la utilidad de la metodología para aumentar la empleabilidad de los estudiantes del grado en publicidad es que en cada una de las dos ediciones, los estudiantes que han participado en el programa y han realizado las prácticas han recibido una oferta de trabajo de los responsables del departamento de recursos humanos de $\mathrm{C}+\mathrm{A}$ (dos estudiantes en la primera edición y uno en la segunda edición) (hay que advertir que, hasta el momento, de la segunda edición solo han podido realizar las prácticas dos estudiantes, ya que los otros dos van a comenzarlas en el próximo mes de octubre). Por ello, podemos deducir también que el programa y la metodología CBL han ayudado a C+A a conseguir la detección de talento y la formación conforme a sus procesos y cultura corporativa.

Todo lo anterior nos permite conectar los resultados obtenidos en nuestro proyecto CBL con experiencias previas similares estudiadas en el presente artículo ya que coincide plenamente con la revisión de la literatura de los casos de éxito precedentes que se ha efectuado en el punto 1.5 de la presente investigación. En dicha revisión, los beneficios destacados que los estudiantes percibieron como resultantes de la participación en programas basados en CBL fueron, liderazgo, acercamiento al mundo profesional, mejora de las habilidades de comunicación oral y escrita, conocimiento más profundo sobre las temáticas tratadas en los retos, aplicación práctica de conceptos y tolerancia a la frustración. Además, CBL se ha mostrado cómo un programa fácilmente aplicable a diferentes áreas de conocimiento y escalable a un gran número de estudiantes y, por lo tanto, con un importante potencial de crecimiento incluso a nivel internacional debido a su implantación en las más importantes universidades del mundo.

A consecuencia del éxito de nuestro programa CBL, la facultad de Ciencias Sociales y de la Comunicación ha implementado y desarrollado un nuevo proyecto denominado Challenge Based School en el cual se da cabida a todos los proyectos basados en CBL en todas y cada una de las áreas de conocimiento de dicha facultad, como ciencias jurídicas

Vivat Academia. Revista de Comunicación. 15 diciembre 2019 /15 marzo 2020, nº 149, 1-24 
Agüero Pérez, M. M.; López Fraile, L. A. y Pérez Expósito J. Challenge Based Learning como modelo de aprendizaje profesionalizante. Caso del programa Universidad Europea con Comunica $+A$.

y políticas, educación y humanidades, empresa, marketing y, por supuesto, comunicación.

Todo lo concluido hasta el momento nos lleva a validar nuestra hipótesis y a inferir que el modelo puede ser replicado a otras entidades y a otros ámbitos de especialización educativa. Por último, decir que ya se trabaja en un plan de mejora de cara a la tercera edición, resultante del análisis realizado en el presente artículo, que incluye aspectos como optimizar la organización de las sesiones de tal manera que no se modifique el horario de clase, perfeccionar la visibilidad del programa e inspeccionar si la participación de los alumnos de primer y segundo curso es una opción adecuada a los objetivos del programa.

\section{REFERENCIAS}

Aalborg University (s. f.). Global-Management.

http://www.en.aau.dk/education/master/operations-innovationmanagement-mscin-tech/specialisations/global-management

Baloian, N., Breuer, H., Hoeksema, K., Hoppe, U. \& Milrad, M. (2004). Implementing the challenge based learning in classroom scenarios. Proceedings of the Symposium on Advanced Technologies in Education, 4.

https://pdfs.semanticscholar.org/f4da/54660cbd763d9d3376aac9c7ba320cb99cde.pd f?_ga $=2.191035561 .263935819 .1571150268-2057572188.1571150268$

Benítez, A. \& García, M. (2013). Un Primer Acercamiento al Docente frente a una Metodología Basada en Proyectos. Formación Universitaria, 6(1), 21-28. Recuperado de https:/ / scielo.conicyt.cl/scielo.php?script=sci_arttext\&pid=S0718$\underline{50062013000100004}$

Boud, D. \& Feletti, G. (1998). The Challenge of Problem-based Learning. London. Kogan Page.

Cancino, R. (2018). El PBL (Project Based Learning): El caso de la Universidad de Aalborg. En A. Villa Sánchez. (Ed.), Tendencias actuales de las transformaciones de las universidades en una nueva sociedad digital (pp. 57-70). Vigo, España: Universidad de Vigo.

Carrión, C. Soler, M. y Avmerich, M. (2015). Análisis de la Validez de Contenido de un Cuestionario de Evaluación del Aprendizaje Basado en Problemas. Un Enfoque Cualitativo. Formación Universitaria, 8(1), 13-22. doi: http://dx.doi.org/10.4067/S0718-50062015000100003

Vivat Academia. Revista de Comunicación. 15 diciembre 2019 /15 marzo 2020, nº 149, 1-24 
Agüero Pérez, M. M.; López Fraile, L. A. y Pérez Expósito J. Challenge Based Learning como modelo de aprendizaje profesionalizante. Caso del programa Universidad Europea con Comunica $+A$.

Cheung, R.; Cohen. J.; Lo, H. \& Elia, F. (2011). Challenge Based Learning in Cybersecurity Education. In Proceedings of the 2011 International Conference on Security Management. world-comp.org. Las Vegas, Nevada, USA.

De los Ríos, I., López, F. \& García, C. (2015). Promoting Professional Project Management Skills in Engineering Higher Education: Project-Based Learning (PBL) Strategy. International Journal of Engineering Education, 31(1), 184-198.

Digital Promise (2017). Powerful Learning. Recuperado de https://digitalpromise.org/wp-content/uploads/2018/08/2017-Digital-PromiseAnnual-Report.pdf

Efrén Mora, L. C. \& González Marrero, A. M. (2013). Real object mapping technologies applied to marine engineering learning process within a CBL methodology. Procedia Computer Science, (25), 406-410. doi: https://doi.org/10.1016/j.procs.2013.11.051

Escamilla, A. (2008). Las competencias básicas. Claves y propuestas para su desarrollo en los centros. Barcelona: Graó.

Gallego Ceide, A.; Terrón López, M. J.; Lagioia, R. \& Valleni, C. (2015). RPAS from cradle to flight: a project based learning experience. Recuperado de http://hdl.handle.net/11268/4218

Hall, C. \& Fernandez, L. A. (2018). UCONN Grad Students Gain Valuable Global Knowledge by Partnering with Henkel. Recuperado de https://www.business.uconn.edu/2018/06/20/uconn-grad-students-gain-valuableglobal-knowledge-by-partnering-with-henkel/\#

Harvard Innovation Lab (2015). President's Challenge Topics. https://ilab.harvard.edu/experientiallearning/presidents-challenge/president $\% \mathrm{E} 2 \% 80 \% 99 \mathrm{~s}-$ challengetopics

Hmelo-Silver, C. E. (2004). Problem-Based Learning: What and How Do Students Learn? Educational Psychology Review, 16(3), 235-266.

Institute of Education Sciences (2010). Effects of problem-based economics on high school economics instruction. Recuperado de https://ies.ed.gov/ncee/edlabs/regions/west/pdf/REL_20104012.pdf

Johnson, L. \& Adams, S. (2011). Challenge based learning: The report from the implementation project. Austin, Texas: The New Media Consortium.

Vivat Academia. Revista de Comunicación. 15 diciembre 2019 /15 marzo 2020, nº 149, 1-24 
Agüero Pérez, M. M.; López Fraile, L. A. y Pérez Expósito J. Challenge Based Learning como modelo de aprendizaje profesionalizante. Caso del programa Universidad Europea con Comunica $+A$.

Johnson, L. \& Brown, S. (2011). Challenge Based Learning: The Report from the Implementation Project. Austin, Texas: The New Media Consortium. Recuperado de https://www.learntechlib.org/p/49837/

McWilliam, E. \& Taylor, P. (2001). Rigorous, Rapid and Relevant: Doctoral Training in New Times. In B. G., TW Maxwell \& Peter Shanahan (Ed.), Doctoral Education and Professional Practice: The Next Generation? (pp. 229-246). Armidale: Kardoorair Press.

Membrillo-Hernández, J. et al. (2018). Challenge-based Learning: The Case of Sustainable Development Engineering at the Tecnologico de Monterrey, Mexico City Campus. International Journal of Engineering Pedagogy, 8(3), 137-144.

MIT (2015). MIT Ideas Global. Challenge. http:/ / globalchallenge.mit.edu/problems

Morales, C. \& Torres, A. (2015). Aprendizaje basado en proyectos para el desarrollo de competencias. Revista Iberoamericana de Producción Académica y Gestión Educativa, (2), 110.

Nilsook, P.; Premsmith, J. \& Wannapiroon, P. (2016).System Design of Challenge-Based Learning Management System. In: The 4th International Conference of technical Education. Faculty of Technical Education, King Mongkut's University of Technology North Bangkok, Bangkok, Thailand.

O'Mahony, T.; Vye, N.; Bransford, J.; Sanders, E.; Stevens, R.; Stephens, R.; Richey, M., Lin, K. \& Soleiman, M. (2012). A Comparison of Lecture-Based and Challenge-Based Learning in a Workplace Setting: Course Designs, Patterns of Interactivity, and Learning Outcomes. Journal of the Learning Sciences, 21(1), 182-206. doi: https://doi.org/10.1080/10508406.2011.611775

Perrenoud, P. (2004). Diez nuevas competencias para enseñar. Barcelona: Graó.

Perrenoud, P. (2012). Cuando la escuela pretende preparar para la vida. Barcelona: Gráo.

Przybysz-Zaremba, M.; Rimkūnienè, D.; Vasilienė-Vasiliauskienė, V. \& Butvilas, T. (2017). Project-based learning: the complexity, benefits and challenges within $21^{\text {st }}$ Century Education. Journal of Educational Review, 10(1-2), 95-99.

Rice, M. \& Shannon, L. (2015). Developing Project Based Learning, Integrated Courses from Two Different Colleges at an Institution of Higher Education: An Overview of the Processes, Challenges, and Lessons Learned. En: EDSIG Conference, Wilmington, North Carolina USA. Recuperado de http:// proc.iscap.info/2015/pdf/3432.pdf

Vivat Academia. Revista de Comunicación. 15 diciembre 2019 /15 marzo 2020, nº 149, 1-24 
Agüero Pérez, M. M.; López Fraile, L. A. y Pérez Expósito J. Challenge Based Learning como modelo de aprendizaje profesionalizante.

Caso del programa Universidad Europea con Comunica $+A$.

Saverv, J. (2006). Overview of problem-based learning: Definitions and distinctions. Interdisciplinarv Journal of Problem-Based Learning, 1(1), 9-20.

Schmidt, R. \& Gibbs, P. (2009). The challenges of work- based learning in the changing context of the European Higher Education Area. European Journal of Education, 44(3), Part 1.399-410.

Swiden, C. (2103). Effects of Challenge Based Learning on student motivation and achievement. (Master thesis). Montana State University, Bozeman Montana.

Terrón López, M. J; Blanco Archilla, M. Y. \& Velasco Quintana, P. (2017). A project based learning experience using NGO Projects and a volunteer program abroad. Recuperado de http://hdl.handle.net/11268/6328

Trujillo Torres, J. M; Hinojo Lucena, M. A.; Marín Marín, J. A.; Romero Díaz de la Guardia, J. J. y Campos Soto, A. (2015). Análisis de experiencias de aprendizajes basados en proyectos prácticas colaborativas B-Learning. edmetic, Revista de Educación Mediática y TIC, 4(1), 51-77.

Velasco Quintana, P.; Gómez de Merodio Perea, M. R. \& Terrón López, M. J. (2017). Learning together - working together. PBL in cooperation with companies. Recuperado de http://hdl.handle.net/11268/6549

Xu, Y. \& Liu, W. Q. (2010). A project-based learning approach a case study in China. Asia Pacific Education Review, (3), 636-370.

Zabala, A. \& Arnau, L. (2007). Como aprender y enseñar competencias. Barcelona: Gráo.

Zavirov, C. (2013). New Challenges for the Project-based learning in the Digital Age. Trakia Journal of Sciences, (3), 298-302.

\section{AUTORES}

\section{María Mercedes Agüero Pérez}

Vicedecana del área de Comunicación de la Facultad de Ciencias Sociales y de la Comunicación. Desde su incorporación en el 2007 ha ejercido distintos cargos de responsabilidad en la Universidad Europea, ha sido Directora Académica de Empresa y Comunicación desde noviembre de 2011 hasta enero de 2016. Coordinadora Académica de la Facultad de Artes y Comunicación desde noviembre de 2009 hasta noviembre de 2011.

Doctora en Comunicación por la Universidad Europea de Madrid, Certificado por la UCLA Anderson School of Management en Women Lidership, Máster en Gestión y

Vivat Academia. Revista de Comunicación. 15 diciembre 2019 /15 marzo 2020, nº 149, 1-24 
Agüero Pérez, M. M.; López Fraile, L. A. y Pérez Expósito J. Challenge Based Learning como modelo de aprendizaje profesionalizante.

Caso del programa Universidad Europea con Comunica $+A$.

Administración de Empresas por la Universidad Nacional de Educación a Distancia y Licenciada en Ciencias de la Información (especialidad en Publicidad y Relaciones Públicas) por la Universidad Complutense de Madrid. Sus principales líneas de investigación están relacionadas con la reputación corporativa, identidad e imagen de marca y redes sociales.

mariamercedes.aguero@universidadeuropea.es

Orcid ID: https:// orcid.org/0000-0001-9412-0772

Google Scholar: http://cort.as/-Sd-0

\section{Luis Antonio López Fraile}

Doctor en Comunicación ("Cum Laude") por la Universidad Europea de Madrid. Licenciado en CC. Políticas y Sociología por la Universidad Complutense de Madrid (especialidades Socio-laboral y Comportamiento del Consumidor) y Master en Dirección de RR.HH. y Organización por ESIC. Ha realizado estancias internacionales en la Università degli studi di Bergamo (Italia). Actualmente es profesor y coordinador de prácticas en la facultad de CC. Sociales y de la Comunicación de la Universidad Europea de Madrid, donde imparte las asignaturas "Gestión de Productos y Marcas", "Estrategias Integradoras", "Laboratorio de Estrategia Publicitaria", "Estrategias Publicitarias", "Gestión y Planificación de Cuentas" y "Dirección de Servicios al Cliente". Fuera del entorno universitario, el profesor López fue director de cuentas, director de RR.HH. y director general en WYSIWYG, Director de Proyectos en MyV Cuadrado y responsable de cuentas en agencias de publicidad, como MacCann, Saathi, Publitext o Matriz.

luis.lopez@universidadeuropea.es

Orcid ID: https:// orcid.org/0000-0002-4997-1815

Google Scholar: https://scholar.google.es/citations?user=fTfSdkoAAAAJ\&hl=es

\section{Javier Pérez Expósito}

Doctor en Comunicación y Marketing y MBA en Business Administration. Más de 10 años de experiencia docente en el área de la estrategia de marketing y planificación estratégica. He desarrollado además diversas responsabilidades de gestión como la dirección de master en Dirección de Comunicación, la coordinación de los Proyectos Fin de Grado del área de Publicidad o la dirección de los laboratorios de Publicidad. Actualmente ocupando el cargo de Coordinación de Titulación del grado en Publicidad. En el ámbito internacional, he impartido clases en universidades americanas como FSU (Florida State University) y en Kendall College Business School (Chicago). En el ámbito investigador, he publicado varios artículos y capítulos de libros sobre la figura de celebridades y su relación con la comunicación, así como la importancia en comunicación política y nuevas tecnologías. Además, también he publicado sobre formación experiencial y desarrollado programas de formación dual en el ámbito de la Publicidad.

javier.perez3@universidadeuropea.es

Orcid ID: https:// orcid.org/0000-0002-5732-8416

Vivat Academia. Revista de Comunicación. 15 diciembre 2019 /15 marzo 2020, nº 149, 1-24 